\title{
Article \\ Retinal Optical Quality of Multifocal Refractive and Monofocal Intraocular Lenses
}

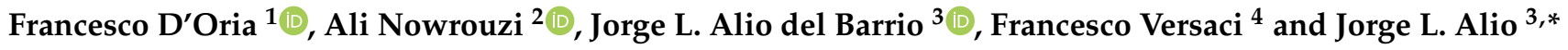 \\ 1 Section of Ophthalmology, Department of Basic Medical Sciences, Neurosciences and Sense Organs, \\ University of Bari, 70124 Bari, Italy; francescodoria91@hotmail.it \\ 2 Ophthalmology Department of Hospital Quironsalud, 29670 Marbella, Spain; alinoroozi_co@yahoo.com \\ 3 Department of Ophthalmology, Miguel Hernandez University, Vissum Miranza, 03015 Alicante, Spain; \\ jorge_alio@hotmail.com \\ 4 R\&D Department, Costruzione Strumenti Oftalmici (CSO), 50018 Florence, Italy; f.versaci@csoitalia.it \\ * Correspondence: jlalio@vissum.com
}

check for

updates

Citation: D'Oria, F.; Nowrouzi, A.; Alio del Barrio, J.L.; Versaci, F.; Alio, J.L. Retinal Optical Quality of Multifocal Refractive and Monofocal Intraocular Lenses. Photonics 2021, 8, 559. https://doi.org/10.3390/ photonics 8120559

Received: 23 August 2021

Accepted: 6 December 2021

Published: 8 December 2021

Publisher's Note: MDPI stays neutral with regard to jurisdictional claims in published maps and institutional affiliations.

Copyright: (c) 2021 by the authors. Licensee MDPI, Basel, Switzerland. This article is an open access article distributed under the terms and conditions of the Creative Commons Attribution (CC BY) license (https:/ / creativecommons.org/licenses/by/ $4.0 /)$.
Abstract: (1) Background: This study aimed to evaluate and compare the clinical optical image quality following implantation with different premium IOLs by the analysis of the point spread function (PSF) Strehl ratio using a Pyramidal WaveFront-based sensor (PWS) aberrometer at two different pupil sizes. (2) Methods: This study included 96 eyes of 70 patients implanted with: (1) 19 AcrySof SA60AT (control group); (2) 24 LENTIS Mplus LS-313 MF30; (3) 33 LENTIS Mplus LS-313 MF15; and (4) 20 Precizon Presbyopic. Main outcome measures were PSF Strehl ratio, PSF Strehl ratio excluding second-order aberrations (PSFw2), total root-mean-square (RMS), and low- and high-order aberrations' RMS measured by PWS aberrometer. Results: SA60AT had the highest significant PSFw2 Strehl ratio at both 3 - and 4-mm pupil size $(0.41 \pm 0.11$ and $0.28 \pm 0.07)$ followed by LENTIS Mplus 15 (group C, $0.35 \pm 0.1$ and $0.21 \pm 0.06$ ) and a near tie between LENTIS MPLUS 30 (group B, $0.27 \pm 0.08$ and $0.18 \pm 0.06$ ) and Precizon Presbyopic (group D, $0.27 \pm 0.07$ and $0.17 \pm 0.04$ ). MPlus MF15 was found to be significantly better than MPlus MF30 at both $3.00 \mathrm{~mm}(p<0.0001)$ and $4.00 \mathrm{~mm}(p=0.002)$. (4) Conclusions: The PSFw2 represents a new tool to objectively evaluate the far distance retinal image quality of multifocal IOLs, and the far distance clinical image quality parameters measured by PWS aberrometer differed significantly according to the technology of the implanted lens.

Keywords: retinal image quality; multifocal refractive IOL; monofocal IOL; pyramidal aberrometer

\section{Introduction}

Cataract is still considered one of the main causes of visual loss worldwide [1,2]. Cataract surgery can be considered not only as a therapeutic but also as a refractive procedure, by the replacement of the natural opacified crystalline lens by an intraocular lens (IOL) or even by the replacement of a transparent crystalline lens by an IOL for pure refractive reasons with consequent long-term implantation and subsequent deterioration. [3] The continuous surgical and technological innovations require new approaches for surgeons to keep in constant contact with the latest technological developments, to offer to the patient the best solution to restore vision.

A new concept introduced in the last years is the selection of the IOL according to its optical and aberrometric characteristics: This aspect is extremely important when considering the use of premium IOLs that offer near and intermediate vision. Any IOL should ideally minimize ocular wavefront (WF) aberrations and optimize the retinal image quality [4] to prevent the impact that a low retinal image can have on visual quality and contrast sensitivity and reduce the risk of neuroadaptation failure [5].

Our research group recently evaluated in vivo the optical quality for a far distance of the eye with different types of premium IOLs based on a multifocal, both refractive and diffractive, extended depth of focus (EDoF), or accommodative optic, using a new pyramidal wavefront-based sensor (PWS) aberrometer. [6] The PWS aberrometer has several 
characteristics that help in the interpretation of the aberrometric data in pseudophakic patients implanted with multifocal IOLs, overcoming the limits of the otherwise commonly used Hartmann-Shack (HS) method, the Tscherning principle, or Ray-tracing [7-9].

The big advantage of a PWS is that the WF is sampled at the very last measuring stage, and this allows for a much higher resolution. For instance, an HS sensor discretizes the wave-front at the lenslet stage and the number of the lens on the lenslet determines the number of measured samples. For HS, usually we have 1000-2000 lenses with a resolution of 250-1250 micron. Osiris, on the contrary, has a resolution of 41 microns $(45,000$ samples at the maximum pupil size) [6].

The present study aimed to more deeply evaluate the retinal image quality of the eye implanted with multifocal refractive IOLs as compared to monofocal spherical IOL.

\section{Materials and Methods}

\subsection{Study Design}

This was a prospective, consecutive, comparative, case series study. Inclusion criteria were uncomplicated cataract or refractive lens exchange surgery. Exclusion criteria included amblyopia, any type of corneal disease or opacity, previous ocular surgery, chronic or recurrent uveitis, acute ocular disease or external/internal infection, diabetes mellitus with retinal changes, and glaucoma. Only eyes with mean pre-operative keratometry between 42.5 and $45 \mathrm{D}$, spherical aberration between $\pm 0.25 \mu \mathrm{m}$, corneal HOA of $<0.5 \mu \mathrm{m}$, and angle kappa of less than $0.4 \mathrm{~mm}$ were included in the study. Cases with intraocular complications that could affect IOL performance or IOL stability were excluded, as well as any capsular changes that would increase the risk of decentration or tilt of the IOL, such as zonular weakness or pseudoexfoliation syndrome [6]. All procedures adhered to the tenets of the Helsinki Declaration of the World Medical Association and were conducted based on of the Ethical Board Committee approval of our institution.

All patients had an evaluation at 3 months postoperatively of the ocular aberrations using the PWS-based aberrometer, Osiris (Costruzione Strumenti Oftalmici, Firenze, Italy) at 3.0- and 4.0-mm pupil diameters. In all cases, three consecutive acquisitions were obtained and the following aberrometric parameters were analyzed: total root-meansquare (RMS), LOA RMS, HOA RMS, and PSF Strehl Ratio. Briefly, as described in our previous study [6], for the measurement of retinal image quality based on PSF Strehl Ratio, the second-order aberrations were eventually excluded in the analysis of the PSF Strehl Ratio (PSFw2) by decomposing the wave-front in terms of Zernike.

\subsection{The Osiris Pyramidal Aberrometry System}

The Osiris aberrometer bases its working principle on a high-resolution, four-faced pyramidal WF sensor, that by comparing the light intensity among four images of the entrance pupil of the patient (known as sub-pupils) we can provide the measurement of the WF and refractive error. The WF error, as well as the refractive error, is sampled with 45,000 points at the maximum pupil, which corresponds to a resolution of $41 \mu \mathrm{m}$. The device uses an extended light-emitting diode source emitting at $850 \mathrm{~nm}$ as a measurement of visible light that would have dramatically reduced the pupil size. The results are then converted to the 585-nm wavelength, correcting the effect of longitudinal chromatic aberration. The software shows the direct outcome of the device and, due to its dense resolution, does not need Zernike polynomial-based modal reconstruction to retrieve the ocular WF shape.

A PWF-based aberrometer measures the slopes of a wave-front, focusing the foveal source image on the top of a pyramidal prism with a large apex angle. The prism is acting to split the beam into four parts creating "sub-pupils". The "sub-pupils" will be identical in the case of aberration-free wave-front and the presence of optical aberrations; the intensity distribution among the pupils will be a function of the first derivative of the wave-front along $\mathrm{x}$ and $\mathrm{y}$. It was demonstrated that for each point the derivative along the $x$-axis is proportional to the difference between left and right sub-pupils, and the one along the 
$y$-axis is proportional to the difference between top and bottom sub-pupils. The wave-front error matrix is finally calculated with a numerical integration method starting from the directional derivatives. Nevertheless, a Zernike fitting is available during the analysis process for back compatibility with previous devices and to split the overall WF error in Zernike main components. [7] The Osiris aberrometer provides accurate and repeatable measures of LOAs and HOAs, even in the challenging cases of the peripheral retina and multifocal optics [9]. The big advantage of a pyramid-based sensor is that the wave-front is sampled at the very last measuring stage and this allows for a much higher resolution.

\subsection{IOLs Studied}

All patients underwent micro incisional cataract surgery. Each patient included in this study was implanted with one of four different IOL, and the IOL power was targeted to emmetropia in all cases: monofocal spherical AcrySof SA60AT as the control group (Alcon, Inc., South Freeway, Fort Worth, TX, USA, group A); multifocal refractive LENTIS Mplus LS-313 MF30 (Oculentis GmbH, Berlin, Germany, group B); multifocal refractive LENTIS Mplus LS-313 MF15 (Oculentis GmbH, Berlin, Germany, group C); and multifocal refractive Precizon Presbyopic (Ophtec BV, Groningen, The Netherlands, group D).

\subsection{Statistical Analysis}

Absolute and relative frequencies were calculated to describe the qualitative variables, whereas means and standard deviations were used for quantitative ones. For time-nondependent variables, Chi-squared test (Pearson or Fisher) and ANOVA were estimated to determine differences between groups. However, for time-dependent variables, paired $t$-tests and mixed linear models were obtained for that purpose. The error type I was fixed in 5\%. To detect a significant difference in the postoperative values of PSFw2, the main outcome, we calculated that 11,14 , and 16 eyes per group would have been necessary to obtain $80 \%, 90 \%$, and $95 \%$ power, respectively. All the calculations were made using IBM SPSS Statistics 25 and R 3.5.1.

\section{Results}

A total of 96 eyes of 70 patients aged between 42 and 81 years $(63 \pm 9.5)$ were included in this study and grouped according to the type of pseudophakic IOL implanted, in four different groups. As shown in Table 1, these patients were well matched in terms of IOL power.

Table 1. Summary of studied groups.

\begin{tabular}{cc}
\hline \multicolumn{1}{c}{ Group } & Mean \pm SD or $\boldsymbol{n}(\mathbf{\%})$ \\
\hline Age, years & $70.4 \pm 7.1$ \\
AcrySof SA60AT & $61.9 \pm 7.8$ \\
LENTIS Mplus LS-313 MF30 & $60.9 \pm 9.2$ \\
LENTIS Mplus LS-313 MF15 & $61.4 \pm 11.9$ \\
Precizon Presbyopic & \\
\hline Gender, female & $9(75.0)$ \\
AcrySof SA60AT & $9(42.9)$ \\
LENTIS Mplus LS-313 MF30 & $14(53.8)$ \\
LENTIS Mplus LS-313 MF15 & $6(60.0)$ \\
Precizon Presbyopic & \\
IOL power, diopter & $20.4 \pm 4.3$ \\
AcrySof SA60AT & $22.1 \pm 3.8$ \\
LENTIS Mplus LS-313 MF30 & $22.5 \pm 3.8$ \\
LENTIS Mplus LS-313 MF15 & $21.4 \pm 2.7$ \\
Precizon Presbyopic &
\end{tabular}

Abbreviation: $n(\%)$, absolute frequency (relative frequency); SD, standard deviation. 
WF aberrations in the far distance image were compared for each of the four lens types at two different pupil sizes ( 3 and $4 \mathrm{~mm}$ ). Total RMS, LOA RMS, HOA RMS, PSF Strehl ratio, and PSFw2 Strehl ratio were recorded and analyzed. Figure 1 shows a sample of PSFw2 obtained with the different lenses included in the study.

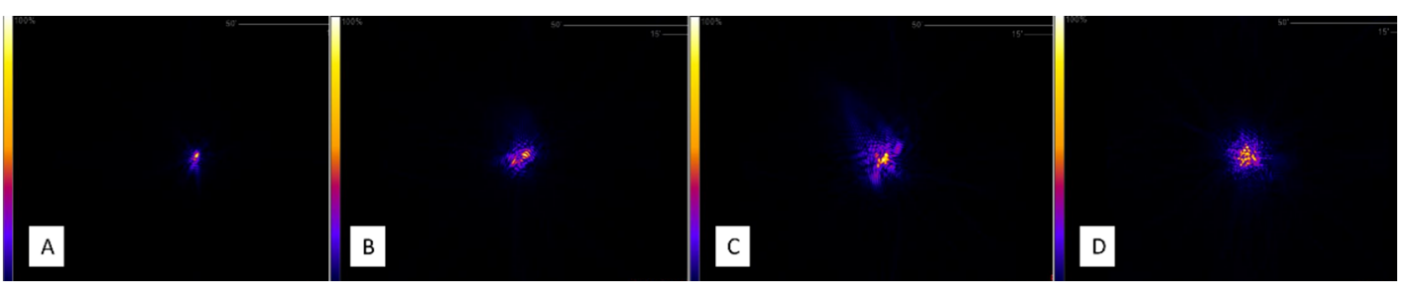

Figure 1. Point Spread Function excluding second-order aberrations (PSFw2) of a sample of patients implanted with different types of intraocular lenses: (A) SA60AT, PSFw2 = 0.5303; (B) MPLUS 15, PSFw2 = 0.3044; (C) MPLUS 30, PSFw2 = 0.2211; (D) Precizon, PSFw2 = 0.2195 .

The overall grouping outcomes of postoperative aberrations of the far distance image with 3.0-mm and 4.0-mm pupil diameters are presented in Table 2.

Table 2. Postoperative values of wave-front aberrations of the analyzed intervention groups at two pupil sizes obtained at 3 months. Abbreviations: HOA, High-order aberration; LOA, low-order aberration; MTF, Modulation transfer function; PSF, Point spread function; PSFw2, Point spread function without second-order aberrations; RMS, root mean square; $\pm \mathrm{s}$, mean \pm standard deviation.

\begin{tabular}{|c|c|c|c|c|c|}
\hline Variable & $\begin{array}{c}\text { AcrySof SA60AT } \\
p \text {-Value } \\
x \pm s\end{array}$ & $\begin{array}{c}\text { LENTIS Mplus } 30 \\
p \text {-Value } \\
\mathrm{x} \pm \mathrm{s}\end{array}$ & $\begin{array}{l}\text { LENTIS Mplus } 15 \\
\begin{array}{c}p \text {-Value } \\
\mathrm{x} \pm \mathrm{s}\end{array}\end{array}$ & $\begin{array}{c}\text { Precizon Presbyopic } \\
p \text {-Value } \\
\mathbf{x} \pm \mathbf{s}\end{array}$ & $\begin{array}{l}p \text {-Value } \\
\text { (Groups) }\end{array}$ \\
\hline $\begin{array}{l}\text { Total RMS } \\
\text { ( } \mu \mathrm{m}):\end{array}$ & $<0.001$ & $<0.001$ & $<0.001$ & $<0.001$ & $<0.001$ \\
\hline $3 \mathrm{~mm}$ & $0.43 \pm 0.19$ & $0.49 \pm 0.18$ & $0.46 \pm 0.15$ & $0.53 \pm 0.17$ & $<0.001$ \\
\hline $4 \mathrm{~mm}$ & $0.66 \pm 0.17$ & $0.83 \pm 0.21$ & $0.83 \pm 0.28$ & $0.78 \pm 0.22$ & $<0.001$ \\
\hline $\begin{array}{l}\text { LOA RMS } \\
\qquad(\mu \mathrm{m}):\end{array}$ & $<0.001$ & $<0.001$ & $<0.001$ & $<0.001$ & $<0.001$ \\
\hline $3 \mathrm{~mm}$ & $0.39 \pm 0.20$ & $0.38 \pm 0.16$ & $0.39 \pm 0.16$ & $0.47 \pm 0.16$ & $<0.001$ \\
\hline $4 \mathrm{~mm}$ & $0.62 \pm 0.20$ & $0.64 \pm 0.25$ & $0.71 \pm 0.32$ & $0.64 \pm 0.21$ & $<0.001$ \\
\hline $\begin{array}{l}\text { HOA RMS } \\
\qquad(\mu \mathrm{m}):\end{array}$ & $<0.001$ & $<0.001$ & $<0.001$ & $<0.001$ & $<0.001$ \\
\hline $3 \mathrm{~mm}$ & $0.16 \pm 0.05$ & $0.30 \pm 0.13$ & $0.21 \pm 0.07$ & $0.25 \pm 0.08$ & $<0.001$ \\
\hline $4 \mathrm{~mm}$ & $0.26 \pm 0.08$ & $0.50 \pm 0.12$ & $0.40 \pm 0.13$ & $0.44 \pm 0.13$ & $<0.001$ \\
\hline $\begin{array}{l}\text { PSF Strehl } \\
\text { Ratio }(\mu \mathrm{m}) \text { : }\end{array}$ & $<0.001$ & $<0.001$ & $<0.001$ & $<0.001$ & $<0.001$ \\
\hline $3 \mathrm{~mm}$ & $0.25 \pm 0.08$ & $0.23 \pm 0.07$ & $0.23 \pm 0.07$ & $0.22 \pm 0.07$ & $<0.001$ \\
\hline $4 \mathrm{~mm}$ & $0.18 \pm 0.05$ & $0.16 \pm 0.05$ & $0.15 \pm 0.04$ & $0.15 \pm 0.04$ & $<0.001$ \\
\hline $\begin{array}{l}\text { PSFw2 Strehl } \\
\text { Ratio }(\mu \mathrm{m}) \text { : }\end{array}$ & $<0.001$ & $<0.001$ & $<0.001$ & $<0.001$ & $<0.001$ \\
\hline $3 \mathrm{~mm}$ & $0.41 \pm 0.11$ & $0.27 \pm 0.08$ & $0.35 \pm 0.10$ & $0.27 \pm 0.07$ & $<0.001$ \\
\hline $4 \mathrm{~mm}$ & $0.28 \pm 0.07$ & $0.18 \pm 0.06$ & $0.21 \pm 0.06$ & $0.17 \pm 0.04$ & $<0.001$ \\
\hline
\end{tabular}

A between-group ANOVA revealed a highly statistically significant difference for all the values at both 3.0 - and $4.0-\mathrm{mm}$ pupil diameters ( $p<0.001$ for all the values). In addition, all the studied aberrations varied significantly as the pupil diameter increased $(p<0.05)$. When looking at the PSFw2 Strehl ratio, SA60AT had the highest significant PSFw2 Strehl ratio at both the 3- and 4-mm pupil sizes (group A, $0.41 \pm 0.11$ and $0.28 \pm 0.07$ ), followed by LENTIS Mplus 15 (group C, $0.35 \pm 0.1$ and $0.21 \pm 0.06$ ) and a near tie between LENTIS MPLUS 30 (group B, $0.27 \pm 0.08$ and $0.18 \pm 0.06$ ) and Precizon Presbyopic (group D, $0.27 \pm 0.07$ and $0.17 \pm 0.04)$. 


\section{Discussion}

IOLs' optics play a fundamental role in the quality of the retinal image: This aspect is becoming increasingly important nowadays, especially with the fall of multifocal IOLs with advanced optics and in the impact that these can have on the postoperative satisfaction and neuroadaptation of the patient [10-12]. Multifocal IOLs are implanted to provide adequate visual performance for far and near in pseudophakic patients; however, they require a process of neuroadaptation that, in some cases, may fail, resulting in patients' dissatisfaction, mainly due to sensations of blurred vision, dysphotopsia, or photic phenomena [5]. Such symptoms constitute a syndrome called neuroadaptation failure, and retinal image quality is of primary concern when considering neuroadaptation [5].

Our study group recently provided an extensive evaluation of postoperative optical effect for far based on PSF Strehl ratio obtained with a PWS-based aberrometer in pseudophakic eyes implanted with different IOLs [6]. This evaluation has never been explored before with all the implanted lenses together. We demonstrated that the Osiris can objectively study the optical quality for far of premium IOLs. In the case of refractive IOLS, the high-order aberrations voluntarily induced by the device explain the multifocality. For refractive or EDoF IOLs, PSF represents how the optical system represents a point at the far distance without any approximation.

In this investigation, we deeply evaluated the optical retinal image for far for three different multifocal refractive IOLs (LENTIS Mplus 15, LENTIS Mplus 30, Precizon Presbyopic), compared to a monofocal spherical IOL commonly used in clinical practice (AcrySof SA60AT).

LENTIS Mplus 15 and LENTIS Mplus 30 are refractive rotational asymmetry lenses, whose design includes an inferior surface-embedded segment with the optical power required for near vision and seamless transitions between the near and far vision zones. There are two different designs of this type of multifocal IOL, a truly bifocal lens with near add of $+3.00 \mathrm{D}$, the Lentis Mplus LS-313 MF30, which provides an acceptable intermediate vision, and the Lentis Mplus LS-313 MF15, which has a near addition power of $1.5 \mathrm{D}$ to provide distance and intermediate visual rehabilitation after cataract surgery with a lower incidence of optical side effects. Its vertical asymmetric optical geometry provides two distant foci for far and near vision by the presence of a calculated magnitude intraocular primary coma [13,14]. Although some amounts of vertical coma have a positive effect on near visual acuity because of the enhanced depth of focus, high values of this aberration could limit the eye's optical quality [15]. As expected, we found a trend toward a larger magnitude of HOA RMS in those eyes implanted with a $3.00 \mathrm{D}$ posterior sector-shaped near-vision zone (Mplus MF30: $0.30 \pm 0.15 \mu \mathrm{m}$ at $3.00 \mathrm{~mm}$ and $0.50 \pm 0.12 \mu \mathrm{m}$ at $4.00 \mathrm{~mm}$ ). These findings suggest that the use of a larger add for the rotationally asymmetric IOL limits the optical quality, with a relative effect on retinal image quality, as expressed by a significantly lower value of PSFw2 Strehl ratio at both pupil diameters $(0.23 \pm 0.07$ at $3.00 \mathrm{~mm}$ and $0.16 \pm 0.05$ at $4.00 \mathrm{~mm} ; p<0.0001)$. We, therefore, compared those patients implanted with IOLs based on similar optics: Among the rotational asymmetric refractive IOLs, LENTIS MPlus MF 15 was found to be significantly better than LENTIS MPlus MF 30 at both $3.00 \mathrm{~mm}(p<0.0001)$ and at $4.00 \mathrm{~mm}(p=0.002)$.

Precizon Presbyopic is a one-piece hybrid hydrophobic and hydrophilic with continuous transition focus that obtains a smoother transition between distance and near vision by combining different sectors in the optical zone of the IOL $[16,17]$. In our study, those eyes presented significantly lower levels of retinal image quality $(0.27 \pm 0.07$ and $0.17 \pm 0.04$, at 3.00 and $4.00 \mathrm{~mm} ; p<0.0001)$ than the monofocal spherical group (SA60AT).

\section{Conclusions}

Learning how the different IOL optics influence the quality of retinal image for far by the study of induced aberrations with the novel PWS-based aberrometer may be considered as a new clinical tool for IOL selection to guide the surgeons in the selection of the IOL that might be implanted according to its optical retinal quality of the image. Monofocal 
aspherical SA60AT provided the highest retinal image quality for far, followed by LENTIS MPLUS 15, LENTIS MPLUS 30, and Precizon Presbiopic. Future prospective studies on this topic are warranted to better understand the relationship between retinal image quality for far as assessed by the study of the PSF Strehl ratio, quality of vision perceived by the patient, and the success of the neuroadaptation process, to compare multifocal lenses among them based on this new tool and to elucidate the retinal image of advanced optics design of multifocal IOLs.

Author Contributions: Conceptualization, methodology, and data curation, F.D., A.N., J.L.A.d.B., F.V. and J.L.A.; Writing—original draft preparation, F.D.; Writing—review and editing, F.D., A.N., J.L.A.d.B., F.V. and J.L.A.; Visualization and supervision, J.L.A.d.B., F.V. and J.L.A. All authors have read and agreed to the published version of the manuscript.

Funding: This study was financed in part by the Network for Cooperative Research in Health "OFTARED", Nodo Dioptrio Ocular, Biobanco Iberia (Reference: RD16/0008/0012). Funded by Instituto de Salud Carlos III and co-funded by the European Regional Development Fund (ERDF), Project "A way to make Europe".

Institutional Review Board Statement: Not applicable.

Informed Consent Statement: Not applicable.

Data Availability Statement: Data are contained within the article.

Conflicts of Interest: The authors declare no conflict of interest.

\section{References}

1. Cedrone, C.; Culasso, F.; Cesareo, M.; Mancino, R.; Ricci, F.; Cupo, G.; Cerulli, L. Prevalence and incidence of age-related cataract in a population sample from Priverno, Italy. Ophthalmic Epidemiol. 1999, 6, 95-103. [CrossRef] [PubMed]

2. Nam, G.E.; Han, K.; Ha, S.G.; Han, B.-D.; Kim, D.H.; Kim, Y.-H.; Cho, K.H.; Park, Y.G.; Ko, B.-J. Relationship between socioeconomic and lifestyle factors and cataracts in Koreans: The Korea National Health and Nutrition Examination Survey 2008-2011. Eye 2015, 29, 913-920. [CrossRef]

3. Alio, J.L.; Arias, A.; D'Oria, F.; Toto, F.; Del Barrio, J.A.; Duarte-Toledo, R.; Artal, P. Light scattering in intraocular lenses explanted 15 to 40 years after surgery. Biomed. Opt. Express 2021, 12, 3485-3494. [CrossRef]

4. Mello, G.R.; Rocha, K.M.; Santhiago, M.R.; Smadja, D.; Krueger, R.R. Applications of wavefront technology. J. Cataract. Refract. Surg. 2012, 38, 1671-1683. [CrossRef]

5. Alio, J.; Pikkel, J. Multifocal Intraocular Lenses: Neuroadaptation. In Multifocal Intraocular Lenses-The Art and the Practice, 1st ed.; Alio, J., Pikkel, J., Eds.; Springer International Publishing: Berlin/Heidelberg, Germany, 2014; pp. 47-52.

6. Alio, J.L.; D'Oria, F.; Toto, F.; Balgos, J.; Palazon, A.; Versaci, F.; del Barrio, J.L.A. Retinal image quality with multifocal, EDoF, and accommodative intraocular lenses as studied by pyramidal aberrometry. Eye Vis. 2021, 8, 37. [CrossRef] [PubMed]

7. Plaza-Puche, A.B.; Salerno, L.C.; Versaci, F.; Romero, D.; Alio, J.L. Clinical evaluation of the repeatability of ocular aberrometry obtained with a new pyramid wavefront sensor. Eur. J. Ophthalmol. 2018, 29, 585-592. [CrossRef]

8. Chamot, S.R.; Dainty, C.; Esposito, S. Adaptive optics for ophthalmic applications using a pyramid wavefront sensor. Opt. Express 2006, 14, 518-526. [CrossRef] [PubMed]

9. Singh, N.K.; Jaskulski, M.; Ramasubramanian, V.; Meyer, D.; Reed, O.; Rickert, M.E.; Bradley, A.; Kollbaum, P.S. Validation of a Clinical Aberrometer Using Pyramidal Wavefront Sensing. Optom. Vis. Sci. 2019, 96, 733-744. [CrossRef] [PubMed]

10. Plaza-Puche, A.B.; Alió, J.L.; MacRae, S.; Zheleznyak, L.; Sala, E.; Yoon, G. Correlating Optical Bench Performance With Clinical Defocus Curves in Varifocal and Trifocal Intraocular Lenses. J. Refract. Surg. 2015, 31, 300-307. [CrossRef]

11. Chang, D.H.; Rocha, K.M. Intraocular lens optics and aberrations. Curr. Opin. Ophthalmol. 2016, 27, 298-303. [CrossRef] [PubMed]

12. Liao, X.; Haung, X.; Lan, C.; Tan, Q.; Wen, B.; Lin, J.; Tian, J. Comprehensive Evaluation of Retinal Image Quality in Comparing Different Aspheric to Spherical Intraocular Lens Implants. Curr. Eye Res. 2019, 44, 1098-1103. [CrossRef] [PubMed]

13. Ramón, M.L.; Piñero, D.P.; Pérez-Cambrodí, R.J. Correlation of Visual Performance With Quality of Life and Intraocular Aberrometric Profile in Patients Implanted With Rotationally Asymmetric Multifocal IOLs. J. Refract. Surg. 2012, $28,93-99$. [CrossRef] [PubMed]

14. Alió, J.L.; Piñero, D.P.; Plaza-Puche, A.B.; Chan, M.J.R. Visual outcomes and optical performance of a monofocal intraocular lens and a new-generation multifocal intraocular lens. J. Cataract. Refract. Surg. 2011, 37, 241-250. [CrossRef] [PubMed]

15. Alfonso, J.F.; Puchades, C.; Fernández-Vega, L.; Montés-Micó, R.; Valcárcel, B.; Ferrer-Blasco, T. Visual acuity comparison of 2 models of bifocal aspheric intraocular lenses. J. Cataract. Refract. Surg. 2009, 35, 672-676. [CrossRef] [PubMed] 
16. Royo, M.; Jiménez, Á.; Piñero, D.P. Clinical outcomes of cataract surgery with implantation of a continuous transitional focus intraocular lens. J. Cataract. Refract. Surg. 2020, 46, 567-572. [CrossRef]

17. Mesa, R.R.; Monteiro, T. Continuous Transitional Focus (CTF): A New Concept in Ophthalmic Surgery. Ophthalmol. Ther. 2018, 7, 223-231. [CrossRef] [PubMed] 